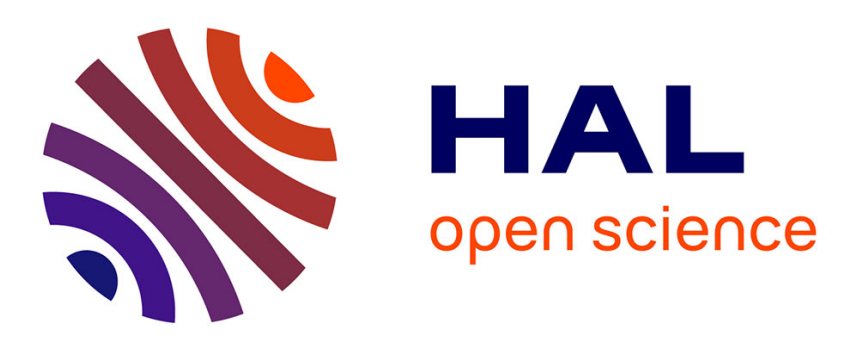

\title{
Limites du modèle délibératif: composer avec différents formats de participation
}

A. Richard-Ferroudji

\section{To cite this version:}

A. Richard-Ferroudji. Limites du modèle délibératif : composer avec différents formats de participation. Politix, 2011, 96 (4), p. 161 - p. 181. 10.3917/pox.096.0161 . hal-00704978

\section{HAL Id: hal-00704978 \\ https://hal.science/hal-00704978}

Submitted on 6 Jun 2012

HAL is a multi-disciplinary open access archive for the deposit and dissemination of scientific research documents, whether they are published or not. The documents may come from teaching and research institutions in France or abroad, or from public or private research centers.
L'archive ouverte pluridisciplinaire HAL, est destinée au dépôt et à la diffusion de documents scientifiques de niveau recherche, publiés ou non, émanant des établissements d'enseignement et de recherche français ou étrangers, des laboratoires publics ou privés. 
Octobre 2011- Proposition d'article pour la Revue Politix - rubrique Varia - Version 2, intégrant les commentaires des relecteurs

\title{
Titre : Limites du modèle délibératif : composer avec différents formats de participation $^{1}$
}

Auteur: Audrey RICHARD-FERROUDJI, Cemagref, UMR G-EAU "Gestion de l'Eau, Acteurs et Usages" 361 rue Jean François Breton BP 509534196 Montpellier Cedex 5 France, Tel. : 0467046354 - Mail : audrey.richard@cemagref.fr

Audrey Richard-Ferroudji est sociologue au Cemagref (UMR G-EAU, Montpellier). En 2008 elle a soutenu une thèse au GSPM (EHESS) sur "l'appropriation des dispositifs de gestion locale et participative de l'eau - Accueillir et composer une pluralité de valeurs, d'objectifs et d'attachements ». Ses travaux de recherche portent sur les équipements politiques de la gestion de l'eau, sur la prise en compte d'une pluralité de rapports à l'environnement et leurs mises en politique selon différentes grammaires.

Résumé : En France la politique de l'eau exige une participation des usagers à sa gestion et une concertation entre les différentes personnes concernées. Les critiques adressées aux dispositifs mis en œuvre pour remplir ces exigences montrent les limites d'une conception uniquement fondée sur le modèle délibératif et invitent à porter un regard attentif aux situations participatives. En nous appuyant sur la théorie des régimes d'engagement, nous proposons une grille de lecture de la manière dont les participants sont engagés et s'engagent dans les dispositifs selon différentes figures de participation : porteur d'enjeu, sujet politique et moral, personne enracinée ou être curieux. Cette approche est mobilisée pour décrire trois formats typiques de participation à la gestion de l'eau, en nous appuyant sur le cas du bassin de l'Orb. Cet article développe une critique interne des dispositifs pour penser leur ajustement. Il invite à penser l'articulation de différents formats de participation pour composer un dispositif approprié et qui reconnaisse les différences entre participants selon plusieurs dimensions.

Title: Limits of the deliberative model : articulating several forms of participation

\begin{abstract}
:
French water policy promotes participative river basin management settings. Most of the settings based on a deliberative model encounter difficulties revealing limits of this model. Following the French so-called "pragmatic sociology", this paper studies the framing of such settings to understand the difficulties encountered. Relying on framework of "regime of engagement", it proposes four figures of participation to describe the way people are engaged and engage themselves: stakeholder, moral subject, rooted person and exploring human being. It discusses three participation formats by describing a case study conducted in the Orb basin, south of France. It paves the way for improved political and social equipment dealing with water by taking into account diverse participation formats. It discusses the very conditions of exploratory engagement, the articulation of justification with other pragmatic regimes and of political models dealing with intimate relations to the river.
\end{abstract}

\section{Texte}

\footnotetext{
${ }^{1}$ Je remercie Rémi Barbier, Olivier Barreteau, Jean-Eudes Beuret, Emmanuelle Cheyns et Laurent Thévenot pour leurs commentaires sur des versions antérieures de ce texte.
} 
En France, la législation sur l'eau exige une participation des usagers à sa gestion et une concertation entre les différentes personnes concernées à l'échelle de territoires définis selon des critères hydrographiques. Les dispositifs proposés pour remplir ces exigences à l'échelle locale se sont multipliés: commissions locales de l'eau, comités de rivière, comités consultatifs, groupes de travail, débats publics, etc. Ils rencontrent souvent des difficultés. Les organisateurs sont déçus du manque de participation ou du résultat des discussions. Réciproquement, des participants ont le sentiment de ne pas pouvoir s'exprimer ou de ne pas être écoutés. Ils dénoncent des réunions manipulatoires ou « qui ne servent à rien ».

Ces critiques ne sont pas nouvelles. De nombreux travaux se sont intéressés à la mise en œuvre de l' « impératif participatif » d'un point de vue analytique, critique ou normatif, en particulier dans le domaine de l'environnement. Une majorité de travaux discute de la participation sous l'angle de l'implication du citoyen dans la vie politique et du pouvoir donné aux participants dans la prise de décision en se fondant sur la critique de la représentation politique classique ou de l'action publique bureaucratique ou technique. L'examen des dispositifs participatifs s'appuie en particulier sur l'explicitation du niveau d'implication des participants, sur une échelle de la participation qui va de l'information à la décision ${ }^{2}$. Les travaux plus récents font un retour critique sur la diversité de démarches mises en œuvre en posant la question de la conceptualisation de la démocratie participative vis-à-vis de la démocratie représentative ou d'un modèle politique délibératif ${ }^{3}$. Selon les critères de bonne délibération un débat doit être public, ouvert, c'est-à-dire égalitaire ou au moins équitable dans l'accès au débat. Les interactions doivent prendre la forme d'une discussion argumentée ouverte à tous les points de vue et orientée vers la production d'un accord raisonné. Le modèle délibératif appuie une approche procédurale de la participation à l'aune de ces critères et préside à la conception ou à l'évaluation de nombreux dispositifs ${ }^{4}$.

Des critiques sont dans le même temps formulées vis-à-vis de l'assimilation de la démocratie participative à la démocratie délibérative. Une critique majeure pointe du doigt l'inégale capacité des personnes et des groupes à peser sur la délibération et à s'y faire entendre ${ }^{5}$. La situation serait rarement celle d'une "discussion libre entre égaux ». La mise en œuvre de démarches participatives ne conduirait alors pas à une égalisation mais à une occultation ou à une reconfiguration des relations de pouvoir. De Leonardis et Pitch montrent qu'un impératif de médiation à «plat» (flat) conduit à cacher les enjeux d'inégalités et rend le pouvoir

\footnotetext{
${ }^{2}$ Parmi les précurseurs Arnstein (S.), « A Ladder of Citizen Participation», Journal of the American Planning Association, 35, 1969.

${ }^{3}$ Cf. par exemple : Blondiaux (L.), Le nouvel esprit de la démocratie Actualité de la démocratie participative, Seuil, 2008. Bacqué (M.-H.), Rey (H.) et Sintomer (Y.), dir, Gestion de proximité et démocratie participative, une perspective comparative, La Découverte, Paris, 2005. Sur le modèle délibératif: Habermas (J.), L'intégration républicaine Essais de théorie politique, Fayard, 1998; Blondiaux (L.), Sintomer (Y.), « Démocratie et Délibération ». Politix, 15(57), 2002 ; Manin (B.), «L'idée de la democratie délibérative dasn la science politique contemporaine Introduction, généalogie et éléments critiques», Politix, 15(57), 2002

${ }^{4}$ Fourniau analyse ainsi les débats organisés par la Commission Nationale de Débat Public ; cf. Fourniau (J.-M.), "Les formes d'organisation du débat et leurs effets dans le processus de décision. Éléments pour une méthodologie d'évaluation des débats organisés par la Commission nationale du débat public », in Mermet (L.), Berlan-Darqué (M.), dir., Environnement : décider autrement. Nouvelles pratiques et nouveaux enjeux de la concertation, Paris, L'Harmattan, 2009.

${ }^{5}$ En soulevant cette limite et en la liant aux différences de culture et de position sociale, Young propose de formuler l'idéal d'une démocratie communicative, plutôt que délibérative, qui accueillerait différents modes de communication en public: l'argumentation, la salutation, la rhétorique et le récit Young (I. $\mathrm{M}$ ), «Communication et altérité. Au-delà de la démocratie délibérative.» in Girard (C.), Le Goff (A.) dir., La Démocratie Délibérative Anthologie de textes fondamentaux, Paris, Hermann, 2010 (1996).
} 
invisible et résistant à sa mise en cause publique ${ }^{6}$. Des personnes refusent alors de s'engager dans les multiples arènes de participation qui leur sont offertes, non pas par incapacité mais parce qu'elles privilégient l'opposition comme modalité de participation ${ }^{7}$. Dans cette perspective, des auteurs revendiquent une « démocratie agonistique » plutôt que délibérative ${ }^{8}$.

D'autres travaux s'attachent à identifier les réussites et les difficultés sans les rabattre sur des ratés et des lenteurs ${ }^{9}$. Il s'agit de sortir d'une approche généralisante et de discuter de l'intérieur les dispositifs de participation mis en œuvre pour dépasser une « double attitude, idéaliste ou cynique ${ }^{10}$ et comprendre le cadre d'interaction qu'ils proposent en prenant au sérieux les techniques et les équipements offerts, tel un site Internet ${ }^{11}$. Le dispositif de participation ou de concertation est devenu ces dernières années l'objet de nombreuses attentions, auxquelles cet article souhaite contribuer en prenant au sérieux les écarts et les résistances à l'impératif participatif qui constitue, dans certains cas, une "nouvelle

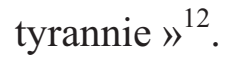

Le terme de dispositif est couramment employé dans le langage administratif pour désigner des moyens mis en œuvre avec une certaine visée : des instruments réglementaires, financiers ou opérationnels. Dans le domaine de l'eau, les Contrats de Rivière ou les SAGE (Schémas d'Aménagement et de Gestion des Eaux) sont des outils standards définis par la loi, utilisés pour satisfaire l'exigence politique de gestion locale et participative de l'eau. Cependant, le terme dispositif peut également être employé dans un sens large pour qualifier un ensemble d'éléments en relation disposés par des êtres humains et susceptibles d'être saisis par eux mêmes ou par d'autres. Les dispositifs de gestion de l'eau sont constitués d'un ensemble de pièces hétérogènes : des documents (un plan de gestion, les statuts d'un organe de concertation, une lettre d'invitation, une lettre d'information), des personnes (un animateur de bassin, un président d'intercommunalité) et des lieux de rencontre (une salle de réunion, les locaux de l'intercommunalité). Être invité à parler à une tribune lors d'une réunion de comité de rivière n'engage pas de la même manière qu'être invité à prendre un café dans les locaux d'un syndicat mixte de bassin ou à discuter au bord d'une rivière. Différents lieux de discussion coexistent sur un territoire. Les dispositifs considérés peuvent avoir été disposés intentionnellement avec une certaine visée, être détournés de leur usage ou être saisis comme une opportunité. Il s'agit de porter un regard dynamique sur les dispositifs et de prêter attention à leurs ajustements en situation, depuis des fonctionnalités initiales jusqu'aux transformations successives par leur usage. S'ils peuvent s'appuyer sur des outils standards ou suivre des guides de bonne pratique en référence au modèle délibératif, les organisateurs et les participants les ajustent. Le terme d'appropriation permet de rendre compte de la variété de transformations des dispositifs pour qu'ils conviennent: dépôt de repères familiers, détournement des fonctionnalités pour satisfaire ses propres objectifs ou définition de

\footnotetext{
${ }^{6}$ De Leonardis (O.), Pitch (T.), « A flat world. The contemporary rhetorics and practices of mediation», à paraître.

${ }^{7}$ Barbier (R.), «Quand le public prend ses distances avec la participation», Natures Sciences Sociétés, 13, 2005.

${ }^{8}$ Blondiaux (L.), Le nouvel esprit de la démocratie Actualité de la démocratie participative, Paris, Seuil, 2008.

${ }^{9}$ Blatrix observe par exemple la trajectoire de débats participatifs, leurs débordements et leurs effets sur les formes de l'action collective Blatrix (C.), « Devoir débattre. Les effets de l'institutionnalisation de la participation sur les formes de l'action collective», Politix, 15 (57), 2002.

${ }^{10}$ Extrait de l'éditorial de «dispositifs participatifs », Politix, 3 (75), 2006 qui propose de développer l'analyse des dispositifs en ouvrant les « boîtes noires » qu'ils représentent.

${ }^{11}$ Benvegnu (N.), « Le débat public en ligne Comment s'équipe la démocratie dialogique ?», Politix, 3 (75), 2006.

${ }^{12}$ Bühler (U.), « Participation 'with Justice and Dignity': Beyond the 'New Tyranny'», Peace, Conflict and Development, 1, 2002.
} 
nouvelles règles pour réguler les échanges. Il ne s'agit donc pas d'aborder la gestion de l'eau et la participation par un projet ou un enjeu mais par les éléments à disposition et susceptibles d'être appropriés par des personnes. Des tables et des chaises sont ainsi à disposition dans les salles de réunion. Elles ont pour fonction de s'asseoir et de poser des documents. Chaque personne peut en faire un usage personnel en déplaçant par exemple une chaise pour se mettre au devant de la scène, ou en retrait pour être plus à l'aise. La disposition des chaises en cercle ou le fait de les aligner face à une tribune fait écho à la différenciation entre démocratie représentative et démocratie délibérative.

Cet article vise à développer une critique interne des dispositifs dans la perspective de penser leurs ajustements. En nous appuyant sur la théorie des régimes d'engagement ${ }^{13}$, nous proposerons dans un premier temps une grille de lecture de la manière dont les participants sont engagés et s'engagent dans les dispositifs selon différentes figures de participation: porteur d'enjeu, sujet politique et moral, personne enracinée ou être curieux. Nous mobiliserons cette approche pour décrire trois formats de participation observés couramment dans le domaine de l'eau comme exemples d'achoppement et de résistance dans la mise en œuvre du modèle délibératif. Nous nous appuierons pour cela sur le cas de l'Orb, un fleuve héraultais ${ }^{14}$. L'analyse de ces résistances nous conduira, en conclusion, à argumenter d'une nécessaire composition de différents formats de participation dans l'ingénierie des dispositifs participatifs.

\section{Comment les participants sont engagés et s'engagent dans les dispositifs participatifs ?}

L'attention aux dispositifs conduit à avoir un regard rapproché sur différentes situations de participation. Il s'agit de qualifier ce que font les dispositifs, ce qui est rendu possible dans certains lieux et ce qui est empêché. Quelles attentes sont formulées vis-à-vis du participant? Comment peut-il se saisir du dispositif ? Cette approche demande de questionner de manière croisée les dispositifs et les dispositions ${ }^{15}$ à participer. Pour cela nous identifions différentes figures de participation endossées, selon la situation, par un participant.

\section{Le porteur d'enjeux ${ }^{16}$ quand le dispositif permet de satisfaire des objectifs contradictoires}

Les usagers ou leurs représentants sont invités dans les dispositifs de gestion de l'eau. Ce faisant, ils sont souvent attendus en tant que porteurs d'enjeux pour défendre des intérêts reconnaissables par les autres participants et liés à leur usage de l'eau. «Ceux qui veulent de l'eau pour arroser les vergers de pêchers » s'opposent à «ceux qui veulent de l'eau pour les poissons » selon la description que nous fait un pêcheur en entretien. Les sciences sociales ont de nombreux outils pour donner à voir l'acteur porteur d'enjeu à travers les catégories d'analyse comme buts, intérêts ou stratégies. Certains auteurs mettent alors l'accent sur

\footnotetext{
${ }^{13}$ Thévenot (L.), L'action au pluriel Sociologie des régimes d'engagement, Paris, La découverte, 2006.

${ }^{14}$ L'article s'appuie sur des enquêtes menées par entretiens et observation de dispositifs. Richard-Ferroudji A., L'appropriation des dispositifs de gestion locale et participative de l'eau - Composer avec une pluralité de valeurs, d'objectifs et d'attachements, thèse pour le doctorat de sociologie, EHESS, 2008.

${ }^{15}$ Elle prolonge ainsi une perspective explorée par exemple par Cochoy (F.), « La captation des publics entre dispositifs et dispositions, ou le petit chaperon rouge revisité ", in F. Cochoy, dir., La captation des publics : "c'est pour mieux te séduire, mon client", Toulouse, Presses Universitaires du Mirail, 2004. Le terme de disposition fait ici référence à la manière dont une personne est bien ou mal disposée lorsqu'elle vient à participer. Il peut renvoyer à une forme de régularité de position sans pour autant être vue selon l'acceptation classique d'un schème incorporé. Les figures de participation sont entendues au sens idéaltypique.

${ }^{16}$ Nous choisissons ce terme en référence à celui de stakeholder mobilisé largement dans la littérature et dans la conception des dispositifs anglophones.
} 
l'engagement stratégique dans les dispositifs de gestion locale de l'eau pour expliquer leur trajectoire ${ }^{17}$. Dans ce type d'engagement, ce qui compte pour la personne c'est d'avoir défini des objectifs à atteindre et d'avoir rassemblé les moyens pour les atteindre lorsqu'elle vient participer dans ce qui est alors vu comme un processus de négociation entre des intérêts contradictoires. Le format de participation proposé doit lui permettre de mettre en œuvre son plan. Les dispositifs semblent bien équipés pour accueillir une telle figure et les participants préparés à l'endosser. Avec une telle perspective, le type d'action possible dans les dispositifs de participation est réduit à celui de satisfaire un objectif lisible. Il est cependant réducteur de ne voir l'action des participants que comme une action stratégique ${ }^{18}$, et d'autres types d'action méritent d'être mis en visibilité.

La théorie des régimes d'engagement propose de distinguer différents régimes pour caractériser l'action en situation au regard du type de «bien » visé par la personne. Thévenot explicite en particulier trois régimes d'engagement: la justification, l'action en plan et l'engagement familier. Un engagement dans l'action en plan vise la satisfaction de l'action accomplie, de l'objectif atteint. Un engagement dans la justification porte la promesse de la satisfaction de contribuer au bien commun et d'être reconnu pour cela, en critiquant ou en justifiant en référence à une certaine conception du bien commun. Dans le régime d'engagement familier, la personne recherche une aise physique ou la satisfaction liée à l'affection pour des choses ou des personnes proches. Chacun des régimes peut être associé à une figure typique de participation. Nous avons associé la figure du porteur d'enjeu à l'engagement en plan. Ce régime d'engagement prend une place centrale dans les dispositifs conçus selon un modèle libéral, au prix d'une réduction dans la façon de considérer la personne et, ce faisant, au risque d'une restriction de la participation voire d'une oppression de l'individu ${ }^{19}$. L'explicitation des autres régimes permet d'éclairer cette réduction.

\section{Le sujet politique et moral quand le dispositif propose de contribuer au bien commun}

Prendre au sérieux différentes catégories d'engagement permet de ne pas rabattre immédiatement la participation sur une stratégie qu'il s'agirait de dévoiler, mais de reconnaître également le participant comme un sujet politique et moral ayant la capacité et la volonté de participer au débat collectif en vue d'un bien commun. Des agriculteurs peuvent s'engager en situation pour la défense de l'intérêt général, de l'environnement ou, plus largement, dans un souci de justice. Les participants peuvent avoir la volonté de contribuer au bien commun et veulent être reconnus pour leur contribution. L'eau, « patrimoine commun de la nation » selon la loi, invite à une discussion et à la justification d'un projet dans une telle perspective. Les moments de débat public visent un tel objectif. Ils ne se limitent pas à favoriser l'expression d'une pluralité d'intérêts. Ils proposent d'accueillir et de mettre en discussion une pluralité de conception d'une bonne gestion de l'eau : celle qui est optimale

\footnotetext{
${ }^{17}$ Sur les objets étudiés ici, cf. par exemple Salles (D.) et Zelem (M.-C.), « Les modalités de la décision publique dans le cadre des politiques de la gestion de l'eau, le cas des contrats de rivière dans le bassin Adour Garonne », in Aspe (C.) et Point (P.) dir, L'eau en représentation, Gestion des milieux aquatiques et représentations sociales, Paris, Quae, 1999; Allain (S.) et Emerit (A.), « Projets d'aménagement hydraulique, concertation et planification participative de bassin : une approche en terme d"'action publique négociée"», Natures Sciences Sociétés, 11, 2003.

${ }^{18} \mathrm{Cf}$. par exemple : Lafaye (C.), « Aménager un littoral. Entre politique et pragmatisme», Etudes rurales, janvierjuin, 1994.

${ }^{19}$ Thévenot (L.), « Sacrifices et bénéfices de l'individu dans un espace public libéral», Cahiers d'éthique sociale et politique, 2008; Cheyns (E.), « Technical rationality and (de)politicisation of standards Multi-stakeholder initiatives in sustainable agriculture», in Gibbon (P.), Ponte (S.) eds., Governing through Standards, London, Palgrave, 2010.
} 
techniquement, celle qui préserve le patrimoine historique, celle qui est égalitaire, etc. Il ne s'agit alors plus de négocier entre des intérêts divergents, mais d'arbitrer ou d'élaborer des compromis entre biens communs visés. Certains auteurs montrent dans l'observation des processus de concertation, la place centrale du travail de légitimation des participants et des dispositifs $^{20}$

L'engagement dans la justification exige de porter une voix détachée, tournée vers l'horizon public d'un bien commun. L'engagement en plan demande de défendre des objectifs lisibles pour les partenaires. Ces actions sont exigeantes et peuvent dissuader de participer. En effet, les personnes ont des dispositions inégales au format requis par l'espace public et celles qui ne sont pas «bien disposées » peuvent être disqualifiées ou exclues ${ }^{21}$. Une charge pèse sur les êtres humains et sur leur vulnérabilité lorsqu'ils doivent passer au format public. Doidy évoque le cas du pêcheur qui a du mal à parler du cours d'eau en général et préfère parler de celui qui lui est familier. Barbier et al. mettent en lumière la charge morale qui pèse sur les personnes invitées à prendre part dans un jury citoyen, dispositifs construits en référence au modèle délibératif ${ }^{22}$. Les dispositifs élaborés selon le modèle délibératif accueillent de façon privilégiée des engagements dans la justification et dans l'action en plan et portent en conséquence les exigences qui leur sont associées. C'est à partir de l'examen de régimes d'engagement familiers, ou dans la proximité, que l'on peut mieux comprendre les difficultés des mouvements requis pour participer à une cause publique et faire entendre sa voix en public.

\section{La personne enracinée quand le dispositif héberge la mise en commun des attachements}

Dans la perspective que nous venons de décrire, l'eau et l'environnement demandent à être traités comme des biens communs en invitant les participants à s'engager en se détachant d'intérêts individuels. Cependant, ils constituent également des objets d'engagement plus personnel. Typiquement, un pêcheur est familier de "son » coin de rivière. Dans un Comité de rivière, il n'est pas invité à parler de cette relation personnelle à l'environnement mais à défendre une utilisation de l'eau pour la pêche. Il réserve l'expression de ces attachements à des moments réservés aux proches, à la marge des réunions par exemple. L'accueil d'engagements familiers entre les participants ou l'expression d'atteintes personnelles sont souvent appréhendés comme des dangers vis-à-vis de l'intérêt général en France. Les opérations de détachement, de dé-singularisation ou de montée en généralité sont des prérequis à la participation. Le détachement correspond à une compétence classiquement demandée à l'homme politique. «Il doit posséder la faculté de laisser les faits agir sur lui dans le recueillement et le calme intérieur de l'âme et par conséquent savoir maintenir à distance les hommes et les choses $»^{23}$. Les représentants associatifs doivent montrer leur capacité à monter en généralité pour être entendus dans le débat public ${ }^{24}$. Le présupposé d'incapacité de détachement conduit à l'exclusion ou à une limitation de la participation. Ainsi, certains dispositifs participatifs vont chercher à enfermer la personne dans son familier et sa particularité. Seul l'habitant est invité à parler et non pas le militant dans les dispositifs de

\footnotetext{
${ }^{20}$ Beuret (J.-E.), La conduite de la concertation pour la gestion de l'environnement et le partage des ressources, Paris, L'harmattan, 2006. Garin (P.), Richard-Ferroudji (A.), « Les conflits de légitimité sous-jacents aux conflits d'usage», La houille blanche, 4, 2008

${ }^{21}$ Doidy (E.), "Faire entendre la voix des usagers dans les concertations environnementales", Sociologie pratique, 7, 2003.

${ }^{22}$ Barbier (R.), Bedu (C.), Buclet (N.), « Portée et limites du dispositif « jury citoyen ». Réflexions à partir du cas de Saint-Brieuc», Politix, 22, 2009.

${ }^{23}$ Weber M., Le savant et le politique, Paris, Plon, 1963 (1919).

${ }^{24}$ Cf. par exemple Lolive (J.), « La montée en généralité pour sortir du Nimby», Politix, 39, 1997.
} 
participation de l'agglomération dunkerquoise $\mathrm{e}^{25}$. Les participants y sont ce faisant dépouillés de toute appartenance collective. Dans d'autres cas, l'enracinement peut être valorisé, de même que les capacités à prendre soin d'un lieu, à s'auto-organiser localement ou à rechercher des arrangements pour accommoder les différents usages du lieu au détriment de l'application d'une règle. Ainsi, Centemeri ${ }^{26}$ analyse les transformations d'un environnementalisme "radical" en un environnementalisme "enraciné" dans le cas de Seveso. Des environnementalistes s'écartent du modèle militant de l'écologie politique pour s'engager dans des actions sociales au plus près des habitants. En Russie, Koveneva observe la composition d'une association de défense de l'environnement qui passe par une mise en commun de proche en proche, veillant à préserver les attachements plutôt, que par une désingularisation de l'environnement local ${ }^{27}$. Ce collectif d' " amis-écologistes » est composé d'habitants qui se soucient de prendre soin, chacun à leur manière, d'une source et qui prennent ainsi en charge ce "site naturel». Les rencontres près de la source ou à domicile sont des situations de communication privilégiées entre les membres de l'amicale et les autres habitants. La communication et le dialogue se fait en deçà du public selon une «grammaire par affinités communes $»^{28}$. Selon cette dernière, la composition de la pluralité ne se fait pas par négociation ou arbitrage, mais par une mise en commun de proche en proche en veillant à préserver les attachements personnels à des lieux communs. La considération du régime d'engagement familier permet de penser la manière dont les dépendances de proximité et les attachements sont reconnus dans une communauté.

\section{L'être curieux quand le dispositif favorise l'exploration}

Enfin, les participants peuvent être engagés dans l'exploration de nouvelles possibilités ou à expérimenter d'autres points de vue sur l'environnement. Ainsi, lorsqu'un représentant de l'agence de l'eau accompagne un agriculteur sur ses parcelles pour comprendre les difficultés d'irrigation, ou lorsque, sur un coin de table, un élu et un représentant associatif discutent de nouveaux projets " en off », ils acceptent de se départir pour un temps de leurs convictions et de ce qu'ils connaissent pour explorer de nouvelles perspectives. Ils tâtonnent. La potentialité du bénéfice collectif de l'exploration invite à proposer des moments qui lui sont dédiés ou qui l'accueillent, alors que la maîtrise et la recherche d'efficacité président souvent à la conception des dispositifs. L'identification d'un régime d'engagement exploratoire ${ }^{29}$ permet de discuter des conditions de possibilité d'une telle exploration. Il permet par exemple de discuter des conditions de réussite de l'ouverture requise par les forums hybrides ${ }^{30}$ attendus pour être le lieu d'une exploration du monde des possibles et de recomposition d'un monde commun. Les exigences du format dialogique (égalité des conditions d'accès au débat, transparence et traçabilité des débats et clarté des règles organisant le débat) peuvent représenter un frein à l'exploration étant donné le risque subversif qui lui est associé. Le mode

\footnotetext{
${ }^{25}$ Lafaye (C.), Flanquart (H.), « La figure de l'habitant et du citoyen dans les dispositifs de participation dunkerquois», Actes du séminaire Cadre de vie, environnement et dynamiques associatives, Paris-La Défense, PUCA-Ministère de l'Equipement, des Transports et du Logement, 2001.

${ }^{26}$ Centemeri (L.), « Retour à Seveso. La complexité morale et politique du dommage à l'environnement », Annales, Histoire, Sciences Sociales, 66(1), 2011

${ }^{27}$ Koveneva (O.), « Les communautés politiques en France et en Russie: regards croisés sur quelques modalités du «vivre ensemble» », Annales, Histoire, Sciences Sociales 66(3), 2011.

${ }^{28}$ Thévenot (L.), « Sacrifices et bénéfices de l'individu dans un espace public libéral», Cahiers d'éthique sociale et politique, 2008.

${ }^{29}$ Auray (N.), « Les technologies de l'information et le régime exploratoire », in Van Andel (P.), Boursier (D.) dir., La sérendipité dans les arts, les sciences et la décision, Paris, Hermann, 2010.

${ }^{30}$ Callon (M.), Lascoumes (P.), Barthes (Y.), Agir dans un monde incertain, Essai sur la démocratie technique, Paris, Seuil, 2001.
} 
d'engagement propre à l'exploration ne s'ordonne pas suivant une grammaire de l'individu doté de volonté, capable de se montrer responsable et de se tenir dans une autonomie, ni d'une personne confiante dans son familier. Il met en valeur la curiosité et la dispersion. Les dispositifs de jeu ou artistiques accordent une place majeure à ce type d'engagement.

La distinction de ces quatre régimes d'engagement, associés à quatre figures de participation, permet de questionner la façon dont les dispositifs engagent les participants sur le moment et dans la durée. Quelles situations permettent l'expression de nouveaux points de vue sans que la personne soit immédiatement sommée de revenir dans les rangs? Quels moments permettent l'expression d'atteintes plus personnelles ? Comment les participants composentils avec celles-ci ? Sous quelles conditions favorables du dispositif, les gens vont traiter autrui en porteur d'enjeux, en sujet politique et moral, en personne enracinée ou en être curieux ? La référence courante à des intérêts ou à des enjeux à représenter ne suffit pas pour appréhender le pluralisme présent dans les dispositifs qui doivent composer avec des biens plus divers dans leur portée. Un agriculteur participant dans un Comité de rivière ne peut être réduit, même si c'est plus commode, à être le défenseur de besoins en eau pour l'irrigation des cultures. Il peut défendre une conception d'une vie bonne sur le bassin ou souhaiter communiquer sur son expérience personnelle de la rivière. Il s'agit de considérer les intérêts des personnes mais aussi leurs ambitions morales, leurs attachements à l'environnement et leur curiosité.

\begin{tabular}{|l|c|c|c|c|}
\hline $\begin{array}{l}\text { Régime } \\
\text { d'engagement }\end{array}$ & Justification & Action en plan & Familier & Exploration \\
\hline $\begin{array}{l}\text { Figure } \\
\text { de participation }\end{array}$ & $\begin{array}{c}\text { Sujet politique } \\
\text { et moral }\end{array}$ & Porteur d'enjeu & $\begin{array}{c}\text { Personne } \\
\text { enracinée }\end{array}$ & Etre curieux \\
\hline Bien engagé & $\begin{array}{c}\text { Contribution au } \\
\text { bien commun }\end{array}$ & Objectif atteint & $\begin{array}{c}\text { Aise ou } \\
\text { affection }\end{array}$ & $\begin{array}{c}\text { Excitation de la } \\
\text { découverte }\end{array}$ \\
\hline
\end{tabular}

Tableau 1 : Quatre régimes d'engagement associés à quatre figures de participation

\section{Trois formats typiques de participation dans le domaine de l'eau}

Chaque dispositif peut favoriser un régime d'engagement ou l'exclure dans ses fonctionnalités ou par son usage. En effet, les participants sont toujours susceptibles de débordements. Ces derniers peuvent conduire à des ajustements ou être disqualifiés, par exemple si l'on « fait comme chez soi » lors d'une réunion publique. Le cadre des régimes d'engagement permet ainsi d'expliquer des tensions et des réussites dans la mise en œuvre de dispositifs de gestion de l'eau ${ }^{31}$ observés dans la vallée de l'Orb, fleuve héraultais.

\section{Le comité de rivière : " grand-messe 》 ou lieu de reconnaissance mutuelle et publique}

Les comités de rivière sont des organes de concertation proposés dans les textes de loi. Le comité de rivière associé au premier contrat de rivière ${ }^{32}$ Orb et au syndicat intercommunal qui le porte était composé de 104 personnes dont 85 représentants de collectivités territoriales, 7 représentants de l'administration et 12 représentants des organismes professionnels et des associations. Ce comité n'a été réuni que deux fois dans le cadre du premier contrat de rivière Orb : à sa signature en 1996 et lors de sa clôture en 2003.

\footnotetext{
${ }^{31}$ Pour une application critique à un dispositif de politique urbaine (les assises de la ville de Bobigny), cf. Charles (J.), Régimes d'engagement et dispositifs participatifs, mémoire de master, Université Catholique de Louvain, Faculté des sciences économiques, sociales et politiques, 2007

${ }^{32}$ Engagement contractuel quinquennal sur des mesures de gestion de l'eau, entre des représentants de l'État, de l'agence de l'eau et de collectivités locales.
} 
La seconde réunion du comité a duré trois heures. Elle a donné lieu à un bilan du premier contrat de rivière ainsi qu'à l'annonce publique d'un accord de principe entre les bailleurs de fonds sur l'élaboration d'un nouveau contrat et la validation des orientations techniques et financières de ce dernier. Cette réunion, organisée par l'équipe du Syndicat Mixte de la Vallée de l'Orb, s'est déroulée en quatre temps : une présentation de l'historique du contrat par le président du syndicat mixte, une présentation des actions accomplies dans les trois thèmes correspondant aux comités consultatifs par les élus responsables de ces comités, une présentation du bilan technique par le directeur du syndicat mixte et un technicien du conseil général, et enfin un temps de discussions. Le cadre ainsi proposé a favorisé une succession de monologues adressés à un auditoire plutôt qu'un débat contradictoire. La réunion a pris place dans une grande salle avec une tribune faisant face à des rangées de tables et de bancs, tel un amphithéâtre universitaire. À la table de la tribune figuraient de "grands » participants : un député, le président du syndicat mixte, le président du conseil général, un sous-préfet et le directeur régional de l'agence de l'eau. Ces derniers ont pris la parole pour de longs discours, alors que la plupart des autres intervenants, hormis ceux qui avaient des présentations programmées, ont posé des questions courtes ou des revendications ciblées.

Le régime de justification a été largement dominant. Le format des informations échangées était public et général. Le cas singulier de l'Orb a été traité en généralité. Lors de la réunion observée, les critiques ont été rares et souvent minimisées ou rapidement évacuées, de même que les propositions novatrices. Les participants ont exprimé leur satisfaction et se sont félicités mutuellement du chemin parcouru. Ils ont valorisé des éléments considérés comme des réussites. Ainsi, le président du conseil général de l'Hérault introduit la réunion en disant : «Dans cet ouest Héraultais, nous sommes dans l'exemplarité de ce qui a été fait». Le président du syndicat mixte a ensuite retracé l'histoire de la gestion du bassin selon une mise en récit valorisante et en insistant sur des dates clé comme celle de la création du syndicat mixte. Plusieurs intervenants, dont des élus, ont lu un texte lors de leur présentation ou se sont appuyés sur un diaporama. Le comité de rivière n'est pas un lieu d'improvisation. Si l'engagement en plan ou des marques de familiarité trouvent leur place, le régime d'engagement exploratoire n'est pas le bienvenu. Les propositions présentées sont celles pour lesquelles il existe un consensus apparent a priori et les revendications exprimées sont «bien connues », comme ne manquent pas de le rappeler certains participants.

Aucune décision n'a été réellement mise au vote au cours de la réunion du comité. À la fin de la réunion, le président du syndicat mixte a sollicité l'accord oral des financeurs sur le projet de second contrat, mais aucune décision n'a été actée. La fonction du comité, d'être un lieu de décision collective, est utilisée uniquement dans le sens d'une validation par consensus apparent d'une décision préparée et débattue dans d'autres lieux, c'est-à-dire telle que personne ne s'y oppose au moment du vote en comité de rivière. Le comité de rivière Orb n'est pas utilisé comme un lieu de débat pluraliste, d'échange discursif de raisons ou de construction des décisions, mais comme un lieu de réaffirmation publique d'un ordre de grandeur des participants et des actions accomplies ou prévues. C'est une forme de rituel ou de cérémonie que l'on retrouve dans la désignation de "grand-messe » utilisée par un représentant associatif. Dans cette logique, à la signature du second contrat de rivière Orb, une « cérémonie de signature » a été privilégiée à l'organisation d'un comité de rivière. Les soucis de légitimité et de reconnaissance président au fonctionnement du comité. En particulier, les participants sont reconnus au titre de leur appartenance au comité, dont la composition cadrée par la règlementation- est longuement débattue avant d'être fixée par arrêté préfectoral. 
Les exigences propres à ce dispositif expliquent le faible nombre de réunions tenues alors qu'une circulaire ministérielle de 2004 demandait un rythme de réunion annuel.

Cette analyse invite à déplacer le regard vers d'autres lieux d'échanges qui accueillent un débat et permettent de "descendre » dans les régimes d'engagement comme y invite un représentant d'usagers en 2005: «En grande réunion, vous n'avancez rien à certains moments. Il faut redescendre ». Le comité syndical fait partie de ces lieux, puisque les élus s'y retrouvent entre eux alors que dans le comité de rivière, ils font face aux représentants des services de l'État et à ceux des usagers. Il n'offre cependant qu'une ouverture limitée à la pluralité et reste dans la logique de la démocratie représentative. Un équipement standard de la démocratie participative qui favorise la descente dans les régimes d'engagement est le comité consultatif.

\section{Le comité consultatif: " défouloir " ou lieu intermédiaire du public pour faire entendre la pluralité}

Au cours de la mise en œuvre du premier contrat de rivière Orb, le syndicat mixte de la vallée de l'Orb a mis en place trois comités consultatifs thématiques, concernant la qualité de l'eau, les inondations et la mise en valeur du fleuve. Alors que le comité de rivière est composé d'un nombre limité de membres dont la représentativité doit être argumentée, dans le comité consultatif, cette exigence est levée au profit d'une ouverture. Onze réunions ont eu lieu en six ans de contrat. L'observation de trois réunions et des entretiens nous permettent de discuter des deux critiques principales formulées à l'encontre de ce dispositif: le manque de « pouvoir » donné aux participants et l'impossibilité de « discuter».

La critique concernant l'impossibilité de discussion est assortie d'une dénonciation du manque de compétence d'autres participants ou de leur engagement dans un format revendicatif. Ces critiques dessinent un format de participation attendu selon lequel le mauvais participant est : «l'intégriste », « l'extrémiste », celui qui « bloque et monopolise les débats», celui «qui reste sur sa position», «l'exalté [qui] casse toute une ambiance positive ». Ceux qui disent «toujours la même chose »sont dénoncés, sans prendre aux sérieux l'attente qu'ils portent dans cette répétition. Les prises de parole n'y formeraient qu'un «brouhaha qui empêche le vrai débat ». À l'inverse, le bon participant serait celui qui est ouvert à la discussion, disposé à l'écoute, à la remise en cause et à faire des concessions. C'est-à-dire celui qui est capable de se mettre au format dialogique ou à entrer dans une négociation. Le fonctionnement du comité est critiqué en référence à un modèle dialogique dont s'écarterait la «culture politique» d'une partie des habitants du territoire, qui auraient «besoin d'un apprentissage de la démocratie». Le comité est alors vu comme un « défouloir » ayant pour fonction de « calmer les gens ».

Une telle considération du comité rejoint la seconde critique formulée à son encontre concernant la limitation de la participation à une consultation, le manque d'écoute des gestionnaires et le manque d'influence de ce qui est dit sur la décision. Le comité consultatif accorde un droit de parole mais ne garantit rien sur ce qui en sera fait si ce n'est l'assurance verbale d'être écouté. L'asymétrie entre participants et gestionnaires est souvent réaffirmée dans la disposition de la salle, avec une table tribune faisant face à des rangées de chaises pour les participants selon une configuration typique de dispositifs de participation observés en France (Photo 1). Un représentant associatif souligne alors qu'il est plus efficace d'aller voir le président du syndicat mixte de bassin dans son bureau, en personne, pour exprimer une doléance plutôt que de revendiquer son point de vue dans un comité consultatif. Il fait ainsi 
référence à la persistance d'un modèle domestique de relations qui rendrait inutile la présence du comité consultatif. Une telle analyse mérite cependant d'être nuancée. Certes, on peut pointer, comme l'ont fait d'autres auteurs pour des dispositifs semblables, le «caractère étriqué de la participation qu'ils encouragent ${ }^{33}$ en termes de contribution à la définition d'un programme d'action, de réalisme et de faisabilité. Dans le même temps, Berger observe une " contribution positive » des voix profanes à la concertation publique en dépassant le constat d'une restriction de la participation. De même, Barthe souligne le « caractère ambivalent des procédures de consultation qui tout en permettant de canaliser les controverses ont pour résultat d'augmenter la "discutabilité" des problèmes et d'ouvrir concrètement l'éventail des choix possibles. ${ }^{34}$ Ce type de moment permet de « rendre discutable » sans veiller à laisser place à une "réelle » discussion. Ainsi, il est intéressant de regarder ce que ce lieu rend possible autant que ce qu'il limite, c'est-à-dire d'expliciter une fonction définie par l'usage.

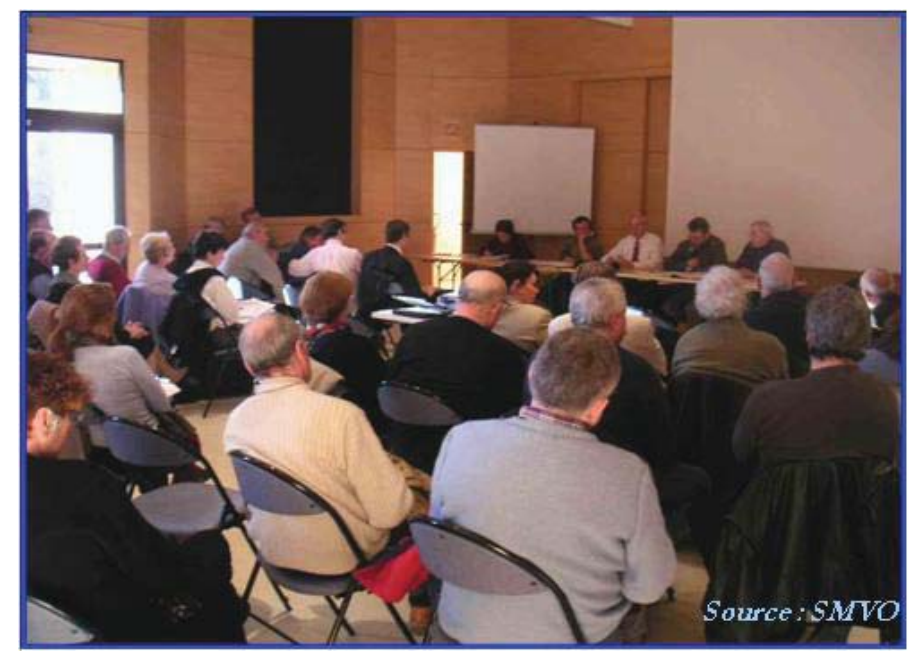

\section{Photo 1 : Exemple typique de dispositif participatif dans le domaine de l'eau et représentatif de l'écart au modèle délibératif, réunion publique, Bédarieux, 2004}

En développant une analyse de son usage à partir du cadre des régimes d'engagement, nous proposons de le qualifier de lieu intermédiaire du public. Le comité consultatif accueille une variété de type d'engagements. Il donne l'occasion d'une expression pluraliste et d'une exploration collective. Son ouverture offre la possibilité intéressante d'une expression de préoccupations de différentes portées. Il est moins exigeant qu'un comité de rivière en termes de " montée en généralité » pour faire entendre sa voix ou de publicité, sans pour autant être le lieu d'un entre soi. Les biens exprimés parcourent une grande palette du proche au public. Il n'exclut aucun régime d'engagement. Il accueille la complainte d'un responsable de camping au risque d'une faillite personnelle, au même titre qu'un débat sur les politiques européennes. Plusieurs participants arrivent à la réunion avec un texte d'intervention préparé et attendent l'occasion de le lire. Ils expriment un sentiment de mission accomplie à la fin de leur lecture. D'autres improvisent au gré des opportunités en émettant parfois des propositions jugées saugrenues par d'autres participants. Ce lieu permet une montée en publicité de la parole des participants, un échange d'informations et le développement d'une connaissance réciproque. En cela, il satisfait certains participants. Le caractère consultatif ne fait pas peser l'exigence de composition de la pluralité exprimée et conduit souvent à une juxtaposition des

\footnotetext{
${ }^{33}$ Berger (M.), « Quand pourrons-nous parler des choses ? ». Quelques contraintes à la référentialité des voix profanes dans un dispositif d'urbanisme participatif », in Cantelli(F.), Roca i Escoda (M.), Stavo-Debauge (J.) Pattaroni (L.), dir., Sensibilités pragmatiques Enquêter sur l'action publique Bruxelles, Berlin, Frankfurt, New York, Oxford, Wien, 2009.

${ }^{34}$ Barthe (Y.), « Rendre discutable. Le traitement politique d'un héritage technologique», Politix, 15, 2002.
} 
paroles. Pour ceux qui revendiquent une plus grande participation, c'est un lieu de mesure réciproque en préparation d'interactions ultérieures. Les participants s'observent et se toisent. Le comité consultatif occupe ainsi une place spécifique dans l'espace de gouvernance, intermédiaire entre des lieux de "débat public» et des lieux en cercle plus restreint qui accueillent la discussion et le «travail» sur des programmes d'action, en particulier les moments qualifiés d'informels. Des personnes interrogées font part de la grâce de tels moments, dont les rencontres au bord de l'eau qui constituent un troisième format typique de participation au sens de prendre part à la gestion de l'eau.

\section{La rencontre au bord de l'eau : se rapprocher de l'objet de discussion, tâtonner et agir}

Un chargé de mission d'une agence de l'eau témoigne lors d'un entretien :

«Souvent, j'ai le sentiment que tu as la réunion de travail et après, dans le repas qu'il peut y avoir, tu arrives parfois même plus à avancer qu'au moment de la réunion. Au moment de la réunion, tu as des discussions sur les thèmes, sur les positions. Au moment du repas, c'est plutôt la phase d'analyse des propositions et à se dire «tiens, éventuellement, il faudrait partir làdessus » [...] Aller ensemble au bord de la rivière ou le repas après, sont des moments cruciaux [...] ça a à voir avec notre société et son fonctionnement. Quand tu te retrouves dans une réunion de comité de pilotage autour d'une table, tu as le sentiment que les personnes sont vraiment plus dans leur rôle, par exemple représentant de l'agence, représentant de l'ASA ${ }^{35}$, etc. $\mathrm{Tu}$ te rends compte que dans d'autres situations autour de ça, tu as certaines barrières ou certains murs qui tombent plus facilement, notamment dans un repas au restaurant ou quand tu vas sur le terrain. [...] Le contexte est différent, tu te retrouves plus face à une personne et les échanges semblent plus aisés. »

Dans ce témoignage, la personne distingue différentes situations. D'une part, il y a les réunions de travail qui donnent lieu à une affirmation de «positions » et dans lesquelles la personne est tenue à un « rôle ». D'autre part, il y a l' « après », le « repas » ou le moment où l'on va « ensemble au bord de la rivière ». Lors des enquêtes, ces moments n'apparaissent pas au premier regard mais se donnent à voir subrepticement. Le comité de rivière est suivi d'un apéritif ; le comité technique d'un repas puis, sur le chemin du retour en voiture, d'un arrêt au bord de l'Orb par plusieurs participants pour voir un problème. Des personnes peuvent s'attarder à la fin d'une réunion pour discuter d'un autre sujet ou " se tenir au courant». Les enquêtés ressentent le besoin d'évoquer ces moments pour expliquer des changements ou des déblocages de situation. Le même technicien de l'agence de l'eau raconte qu'une situation s'est débloquée le jour où il est allé voir les canaux et les terres irriguées avec un président de canal. Ces moments sont souvent qualifiés d' "informels» dans les travaux de sciences sociales $^{36}$ en soulignant leur intérêt mais aussi leur caractère exclusif. Les qualifier d'informels conduit cependant à les considérer comme une boîte noire. Bien que non formalisés, les moments informels ne sont pas informes et revêtent une richesse de formats différents. Les rencontres au bord de l'eau rapportées par les enquêtés correspondent parfois à une saisie d'opportunité, d'autres fois, elles sont préparées voire programmées.

\footnotetext{
35 Association Syndicale Autorisée, regroupant des propriétaires fonciers ayant un droit d'irrigation sur un réseau.

${ }^{36}$ Dans les travaux précurseurs de la sociologie des organisations par exemple, Selznik analyse le décalage entre les buts affichés par une organisation de gestion de l'eau et son fonctionnement réel en soulignant l'importance de l'informel ; cf. Selznick (P.), TVA and the grass roots, Berkeley, University of California press, 1949. Lee reprend les critiques actuelles adressées aux dispositifs de participation pour mettre en valeur les moments informels ; cf. Lee (C.), « Is There a Place for Private Conversation in Public Dialogue? Comparing Stakeholder Assessments of Informal Communication in Collaborative Regional Planning», American Journal of Sociology, 113,2007
} 
Le cadre des régimes d'engagement permet de pointer les spécificités des discussions qui prennent place lors d'une rencontre au bord de l'eau pour comprendre en quoi elles sont considérées comme plus « vraies » que d'autres, comme les qualifiait un directeur de syndicat mixte. Il s'agit de comprendre quelles exigences elles portent et ce qu'elles rendent possible. En particulier, les témoignages contredisent une analyse qui en ferait uniquement ou de façon privilégiée un moment d'engagement stratégique. La rencontre informelle est certes un moment de négociation mais elle donne la place à des ajustements plus riches. Elle accueille des gestes de familiarité, l'expression d'atteintes plus personnelles, des propos moins construits ou moins affirmatifs. Elle peut alléger l'épreuve de jugement et permet d'explorer de nouvelles solutions ou l'expression de points de vue novateurs, comme le souligne l'expression «Tiens éventuellement,... » dans le témoignage précédent. Ainsi, vis-à-vis de moments plus publics, une place privilégiée est accordée aux engagements familiers et exploratoires. Elle donne l'opportunité d'une participation « en personne ». Par la visite sur le terrain ou le repas pris en commun, l'interlocuteur prend de la consistance. Il n'est plus seulement considéré en statut mais en personne. Il peut exprimer sa relation personnelle et singulière avec la rivière. Aller sur le terrain avec quelqu'un implique de se frotter à ses difficultés quotidiennes et permet de comprendre ses soucis et ce à quoi il est attaché. Cela permet de découvrir à tâtons l'autre et l'environnement.

Certains n'acceptent pas de se prêter à ce format d'interaction, et en particulier les personnes soucieuses de maintenir un détachement. "Comprendre les soucis de l'autre » peut être assimilé à «faire preuve d'empathie » ou à «faire des arrangements avec les règles ». Un représentant de l'État peut être réticent à une confrontation physique avec les cas traités dans ses dossiers, de peur que la sollicitude s'impose sur l'application de la règle ou que la complexité observée remette en cause la représentation simplifiée du cas nécessaire à son administration. L'horizon d'un jugement moral n'est cependant pas exclu. Des rencontres au bord de l'eau peuvent être sollicitées dans un souci de justification et de mise en public. Alors que la rivière dans laquelle il prélève de l'eau est à sec en août 2005, un président d'association d'irriguants (ASA) des Pyrénées-Orientales invite les partenaires institutionnels à venir la voir pour comprendre la situation. La visite de terrain est alors appelée pour faire la preuve de la bonne volonté du président suite à une mise en cause de sa parole par un maire. En l'absence d'instruments de mesure du débit accepté collectivement, il convenait d'aller voir la rivière. Dans cette situation, l'engagement en plan (pour obtenir de l'eau pour les agriculteurs) et la justification (du bien-fondé de son action) occupent une place importante. Le président de l'ASA en invitant les partenaires au bord de la rivière a reconnu la critique qui lui était faite de prendre toute l'eau. Cependant, il a souhaité se justifier de ce captage par le faible niveau d'eau et en faire la preuve en conduisant les partenaires sur les berges. Les participants sont mis en présence de l'objet de discussion. Cette mise en présence met à l'épreuve la représentation qui en est faite pour une reconnaissance collective de la pénurie d'eau. Elle permet en outre d'éviter une mise en mots difficile en montrant plutôt qu'en disant. Ainsi, certaines personnes, tel un gérant d'association de canyoning rencontré sur un autre bassin, privilégient cette modalité de coordination en disqualifiant «les gens qui pondent des textes et des arrangements et qui ne sont pas sur le terrain, qui ne rencontrent pas les gens de terrain ». Il dénonce le «blabla », c'est-à-dire une mise en mots nécessairement réductrice. Il privilégie l'action. Dans une rencontre en face à face, au bord de l'eau, les problèmes se résolvent, non pas en verbalisant un accord, mais en faisant voir « comment on fait » pour s'accommoder mutuellement de la présence physique de l'autre et de la rivière. On retrouve ici l'orientation des environnementalistes enracinés de Séveso vers l'action locale et 
la prise de soin d'un environnement qui les entoure ${ }^{37}$. Participer à la gestion de l'eau est alors entendu au sens d'agir sur l'environnement, c'est-à-dire selon une acception de la participation plus large que celle qui préside à la conception des dispositifs participatifs.

\section{Que nous enseignent les écarts au modèle délibératif? Retour sur l'ingénierie des équipements politiques pour la gestion de l'eau}

À partir du cas de l'Orb, nous avons discuté des fonctions et des usages de dispositifs en explicitant les types d'engagement des participants qui y prennent place (tableau 1). La description comparative de trois formats typiques de participation à la gestion locale de l'eau permet d'expliciter des écarts aux attentes, et même des résistances aux formats proposés. Le comité de rivière et le comité consultatif, qui affirment une ambition de participation des usagers et la référence dans leur conception aux principes dialogiques, sont souvent disqualifiés respectivement de "grand-messe » et de " défouloir ». Ils montrent cependant leur pertinence dans un objectif de confirmation publique de décisions ou de reconnaissance collective, pour l'un, et comme «lieu intermédiaire du public» propice à une expression plurielle pour l'autre. Les participants mobilisent d'autres moments pour dialoguer. Les réunions de comité syndical - qui n'ont pas été décrites ici - allègent l'épreuve du public pour les élus dans un moment d'entre soi. De même, des réunions de comités techniques privilégient un travail collectif entre « spécialistes » qui partagent une base de connaissances et des soucis communs. Ces deux comités répondent plus facilement à l'objectif de mettre les personnes qui y participent sur un même plan, mais cela passe par une fermeture assumée et argumentée. Hors dispositif formalisé, la rencontre au bord de l'eau montre sa pertinence pour éprouver en commun l'objet de gestion, avant sa mise en forme collective dans un document : le contrat de rivière. Ce moment donne l'occasion d'une expression de troubles personnels et de points de vue que la personne peine à mettre en forme pour une expression publique. Il permet d'explorer d'autres préoccupations. Des dialogues au bord de l'eau en présence de l'objet de discussion mettent à l'épreuve le discours et permettent de montrer plutôt que de dire lorsqu'il y a un désaccord sur l'état de l'environnement. D'autre part, ils accueillent potentiellement une participation à la gestion de l'eau au sens d'une action sur l'environnement.

\begin{tabular}{|c|c|c|c|c|}
\hline Dispositif & $\begin{array}{c}\text { Présentation } \\
\text { officielle }\end{array}$ & Critique typique & $\begin{array}{c}\text { Régimes } \\
\text { d'engagement }\end{array}$ & Analyse \\
\hline $\begin{array}{c}\text { Comité de } \\
\text { rivière }\end{array}$ & $\begin{array}{c}\text { Décision } \\
\text { Vote le contrat } \\
\text { de rivière }\end{array}$ & « Grand-messe » & $\begin{array}{c}\text { Justification et } \\
\text { action en plan }\end{array}$ & $\begin{array}{c}\text { Reconnaissance es- } \\
\text { qualités Confirmation } \\
\text { des ordres de grandeur }\end{array}$ \\
\hline $\begin{array}{c}\text { Comité } \\
\text { consultatif }\end{array}$ & $\begin{array}{c}\text { Consultation } \\
\text { Ouverture }\end{array}$ & «Défouloir » & $\begin{array}{c}\text { Ouvert à tous } \\
\text { les régimes }\end{array}$ & $\begin{array}{c}\text { Lieu intermédiaire } \\
\text { vers le public }\end{array}$ \\
\hline $\begin{array}{c}\text { Rencontre au } \\
\text { bord de l'eau }\end{array}$ & $\begin{array}{c}\text { Aller voir } \\
\text { Rencontre } \\
\text { informelle }\end{array}$ & $\begin{array}{c}\text { Lieu de } \\
\text { "passe droit } \\
\text { «Entre-soi } »\end{array}$ & $\begin{array}{c}\text { Favorise } \\
\text { exploration et } \\
\text { familier }\end{array}$ & $\begin{array}{c}\text { Epreuve en présence } \\
\text { de ce dont on parle } \\
\text { Mise en commun des } \\
\text { attachements }\end{array}$ \\
\hline
\end{tabular}

Tableau 2 : Analyse comparative de dispositifs de participation à la gestion de l'eau

Le modèle des régimes d'engagement permet de problématiser les exigences de passages des acteurs dans plusieurs régimes, de la plus étroite proximité avec un environnement familier (le pêcheur et son coin de pêche) à l'agir dans l'espace public (un représentant des pêcheurs dans

\footnotetext{
${ }^{37}$ Centemeri, op.cit.
} 
un comité de rivière). Il permet de désigner les exigences tenant à ces passages. Les dispositifs participatifs conçus en référence au modèle délibératif privilégient par construction des engagements en plan ou dans la justification. Ce faisant, et alors qu'ils revendiquent une ouverture, ils opèrent une sélection dans les formes de participation possibles et exigent une mise au format en amont de la participation. Cette exigence doit être prise au sérieux ainsi que l'exclusion d'autres types d'engagement. Certaines paroles doivent prendre le temps de se construire patiemment. Dans la pratique, les participants tâtonnent souvent entre différentes propositions qui doivent gagner en consistance. Ils testent leurs propositions auprès de proches, dans des discussions par téléphone ou avant une réunion, avant de les faire valoir en public. Une lecture de l'appropriation de dispositifs affichés comme participatifs montre des détournements opérés qui ne doivent pas être immédiatement interprétés comme des échecs. Les résistances soulèvent en particulier deux enjeux pour l'ingénierie des équipements politiques pour la gestion de l'eau, concernant la façon de considérer les différences entre participants et l'articulation de différents formats de participation.

\section{Reconnaître les différences entre participants selon plusieurs dimensions}

De nombreux modèles courants de démocratie participative recherchent normativement l'égalité des participants qui entrent en jeu sur un même plan. Mettre sur un pied d'égalité autour d'une table des personnes, tels des élus et des usagers, ne fait qu'éluder les différences de pouvoir, irréductibles selon le sujet traité, et conduit au cynisme des participants. L'égalité parfaite de participation ${ }^{38}$ a un caractère irréaliste qui incite à fixer un autre horizon d'évaluation pour ne pas immédiatement disqualifier une démarche participative sous prétexte qu'il n'y a pas égale participation ou pouvoir égal. L'approche pragmatique invite à prendre au sérieux les différences entre les participants, sans les rabattre uniquement sur des différences d'intérêts ou d'objectifs. L'observation du cas de l'Orb montre la prégnance d'une coordination selon la « grammaire des grandeurs » ${ }^{39}$ avec une visée de bien commun dans la gestion de l'eau. La critique des dispositifs de participation ne passe alors pas par une dénonciation d'inégalité entre les participants, mais par une mise en cause de l'absence de justification des différences ou de l'impossibilité de mettre à l'épreuve les ordres de grandeur existants. La théorie de la justification, que prolonge le régime d'engagement éponyme, met l'accent sur le caractère substantiel, et pas seulement procédural, de la légitimation sur laquelle l'approche délibérative insiste. Cette perspective soumet l'ingénierie des équipements politiques pour la gestion de l'eau à certaines exigences. La pluralité de perspectives de jugement sur l'ordre de grandeur invite à proposer dans les dispositifs différents instruments de mesure et de qualification. Les instruments et les mesures collectivement acceptés sont par exemple reconnus dans un document contrat de rivière. L'inclusion de nouvelles revendications demande la construction de nouveaux équipements de mise à l'épreuve et de légitimation. La dimension temporelle du processus de participation est alors mise en avant. Elle s'exprime, d'une part, dans la nécessité d'un ajustement des dispositifs pour répondre aux critiques. D'autre part, des résistances s'expriment envers un dispositif qui ferait table rase du passé et ne reconnaîtrait pas les différences de contribution en mettant tous les participants sur un même plan. Elles invitent à penser les dispositifs dans la continuité ou en rupture du travail de mise en commun opéré par le passé, alors même que celui-ci mérite d'être remis sur l'ouvrage face aux critiques pointant l'exclusion. En termes d'ingénierie de dispositif, cela signifie par exemple de prêter attention au lieu de rencontre choisi qui n'est jamais neutre ou à

\footnotetext{
${ }^{38}$ Dont le dispositif paradigmatique est le chronomètre mesurant le temps de parole pour une égalisation, utilisé dans des débats dans le cadre de la Commission Nationale du Débat Public.

${ }^{39}$ Boltanski (L.), Thévenot (L.), De la justification. Les économies de la grandeur, Paris, Gallimard, 1991.
} 
faire preuve de précautions lors de l'invitation à participer ${ }^{40}$. Dans ce sens, l'analyse du cas de l'Orb contribue aux travaux qui questionnent le traitement des attachements dans la composition d'une communauté ${ }^{41}$. Il invite à questionner l'enracinement des dispositifs dans les espaces et chez les personnes. Le vocabulaire mobilisé pour qualifier cet enracinement, en termes de gestion domestique ou paternaliste, ne rend pas suffisamment compte des enjeux. La question de la prise en compte des attachements dans la composition d'une communauté est une question politique majeure mise en relief dans le domaine de l'environnement ${ }^{42}$.

\section{Articuler différents formats de participation en accordant une place aux "petites concertations » et à l'exploration curieuse}

Le cadre des régimes d'engagement propose d'évaluer les dispositifs participatifs sur la manière dont ils accueillent et composent avec une pluralité de valeurs, d'objectifs et d'attachements vis-à-vis de l'environnement. La distinction des différents niveaux de biens pour la personne permet de pointer les limites du format délibératif et de penser l'articulation des moments délibératifs avec d'autres formats de participation. Le travail de «mise en politique ${ }^{43}$ de l'eau invite à penser l'articulation de différents formats de participation. Audelà, suivre les participants dans différentes situations nous invite à avoir une perspective plus large sur ce que signifie participer en démocratie : argumenter, mais aussi explorer, faire partager ses attachements, voire prendre soin d'un environnement commun. L'enjeu démocratique n'est pas la participation en tant que telle, mais la composition d'une communauté selon différentes grammaires pour faire ensemble avec une eau qui fait problème. De nouvelles perspectives de recherche émergent de ce changement de regard sur la participation. L'étude de cas présentée invite à prêter attention, d'une part, aux personnes qui portent le travail de composition du commun et, d'autre part, aux formats de participation laissés dans l'ombre. Nous avons ainsi observé des personnes, au cœur des dispositifs de gestion locale de l'eau, travailler activement à ce qui résiste: les animateurs de bassin versant $^{44}$. Ce sont des passeurs. Ils composent avec les dispositions diverses des personnes impliquées, en développant l'art d'ajuster les dispositifs aux situations et aux participants. D'autre part, il s'agit de développer l'analyse comparative de différents formats de participation. Peu de travaux s'intéressent aux «petites concertations » ${ }^{45}$ et aux dispositifs qu'elles mobilisent. De même, une attention plus soutenue mériterait d'être portée aux dispositifs qui accordent, par un cadrage volontaire, une place à l'exploration et à la créativité. La sollicitation de tels dispositifs se multiplie: jeux, démarches prospectives, événements

\footnotetext{
${ }^{40}$ Pour une discussion du rôle de la lettre d'invitation, une petite pièce de dispositif, huile ou grain de sable dans l'engrenage de la concertation, cf. Richard-Ferroudji, op. cit.

${ }^{41}$ Breviglieri (M.), « Penser la dignité sans parler le langage de la capacité à agir », in Payet (J.-P.), Battegay (A.) dir., La reconnaissance à l'épreuve. Explorations socio-anthropologiques, Lille, Presses Universitaires du Septentrion, 2008, Centemeri, « Retour à Seveso. La complexité morale et politique du dommage à l'environnement », art. cité, Thévenot, « Sacrifices et bénéfices de l'individu dans un espace public libéral», art. cité.

${ }^{42}$ Le terme de composition est souvent mobilisé dans les travaux sur le pluralisme. En participant à ce mouvement, nous questionnons ici la prise en compte des attachements entendus au sens des liens construits dans l'engagement familier avec les choses et les personnes. Latour mobilise ce terme dans un sens plus large pour qualifier tout type de lien qui entre dans la composition. Latour (B.), «Factures/fractures. De la notion de réseau à celle d'attachement », in Micoud (A.), Peroni (M.) dir., Ce qui nous relie., Editions de l'Aube, La Tour d'Aigues, 2000

${ }^{43}$ À la suite de Barthe, op cit.

${ }^{44}$ Richard-Ferroudji (A.), «L'animateur de bassin versant: Insuffler vie à une communauté de l'eau », in Gramaglia (C.) dir, Cosmopolitiques, 17, l'eau : un bien commun à composer, 2008.

${ }^{45}$ Pour une analyse et une mise en valeur des «petites concertations », cf. Beuret (J.-E.), Cadoret (A.), Gérer ensemble les territoires Vers une démocratie coopérative, Paris, ECLM, 2010.
} 
Author-produced version of the article published in Politix, 2011, 96(4), 161-181.

The original publication is available at http://www.cairn.info/revue-politix

doi : $10.3917 /$ pox.096.0161

artistiques, théâtre forum, techniques d'animation qui invitent les participants à « sortir de leur position », etc. 